Review Article

\title{
The Prominent Role of HMGA Proteins in the Early Management of Gastrointestinal Cancers
}

\author{
Nathalia Meireles Da Costa, ${ }^{1}$ Luis Felipe Ribeiro Pinto, ${ }^{1}$ Luiz Eurico Nasciutti $\mathbb{D}^{2}$ \\ and Antonio Palumbo Jr. (1) ${ }^{2}$ \\ ${ }^{1}$ Programa de Carcinogênese Molecular, Instituto Nacional de Câncer José de Alencar Gomes da Silva, Rio de Janeiro, RJ, Brazil \\ ${ }^{2}$ Laboratório de Interações Celulares, Instituto de Ciências Biomédicas, Universidade Federal do Rio de Janeiro, \\ Rio de Janeiro, RJ, Brazil \\ Correspondence should be addressed to Antonio Palumbo Jr.; palumbo@icb.ufrj.br
}

Received 26 July 2019; Accepted 23 September 2019; Published 13 October 2019

Guest Editor: Qiang Tong

Copyright ( $) 2019$ Nathalia Meireles Da Costa et al. This is an open access article distributed under the Creative Commons Attribution License, which permits unrestricted use, distribution, and reproduction in any medium, provided the original work is properly cited.

\begin{abstract}
GI tumors represent a heterogeneous group of neoplasms concerning their natural history and molecular alterations harbored. Nevertheless, these tumors share very high incidence and mortality rates worldwide and patients' poor prognosis. Therefore, the identification of specific biomarkers could increase the development of personalized medicine, in order to improve GI cancer management. In this sense, HMGA family members (HMGA1 and HMGA2) comprise an important group of genes involved in the genesis and progression of malignant tumors. Additionally, it has also been reported that HMGA1 and HMGA2 display an important role in the detection and progression of GI tumors. In this way, HMGA family members could be used as reliable biomarkers able to efficiently track not only the tumor per se but also the main risk conditions related with their development of GI cancers in the future. Finally, it shall be a promising option to revert the current scenario, once HMGA genes and proteins could represent a convergence point in the complex landscape of GI tumors.
\end{abstract}

\section{Introduction}

Cancer represents one of the most challenging diseases since the last century, and the exponential growing in the knowledge of its molecular basis shall represent a singular opportunity to translate this knowledge in practical tools, able to effectively impact on life quality of the people affected by this malignancy. This hope mainly resides on the potential application of the recent cellular and molecular discoveries in oncology field, into better strategies for disease prevention, early detection, prognosis increment, and new therapeutic approaches [1]. In fact, the identification of cancer-specific biomarkers has revolutionized this disease management, by increasing the development of personalized medicine, besides changing the "deadly" paradigm commonly associated with cancer [2]. In this sense, HMGA family members (HMGA1 and HMGA2) comprise an important group of genes involved in cancer genesis and progression [3]. Additionally, it has also been reported that HMGA1 and HMGA2 possess an important biomarker potential for the detection and progression of gastrointestinal (GI) tumors [4-8]. GI tumors present a very distinct biology that reflects a myriad of differences in the etiopathology of these malignances, which includes embryonic origin, tissue architecture, and tissue renewal pattern, as well as different etiological factors [9]. In addition, a relevant heterogeneity is observed among GI cancers, concerning clinical aspects, such as the differences harbored in their detection and development [10-12]. For instance, the welldelineated molecular and histopathologic panorama observed for colorectal cancer development, from the premalignant lesion to invasive carcinomas, is not shared by esophageal and gastric tumors. On the other hand, the late diagnosis and high lethality rates observed for esophageal and gastric tumors do not characterize colorectal tumors [9]. Therefore, the search for reliable approaches able to 
efficiently track not only the tumor per se but also the main risk conditions related with their development could represent a great improvement for the management of GI cancers in the future.

\section{HMGA Proteins}

The high mobility group A (HMGA) comprises a protein family of small nonhistone chromatin factors involved in the regulation of gene transcription, acting through either enhancement or suppression of the activity of transcription factors, by remodeling the chromatin structure and orchestrating the recruitment of multiprotein complexes of transcription factors [13]. The HMGA protein family is composed by four members, which are encoded by two genes, HMGA1 and HMGA2. HMGA1 generates three transcript variants- $H M G A 1 a, H M G A 1 b$, and $H M G A 1 c-$ whereas HMGA2 encodes only one transcript-HMGA2 [13]. HMGA family members are characterized by the repetition of three amino acid sequences, called "AT hooks" motifs, which bind preferentially to the minor groove of ATrich sequences in the DNA [14]. Despite the fact that HMGA proteins do not behave as classical transcription factors due to the absence of an intrinsic transcriptional activity, HMGA1 and HMGA2 are capable of modulating the transcription of target genes by inducing alterations in the chromatin structure [15]. Concerning HMGA gene and protein expression patterns, they are significantly expressed during embryogenesis, whereas their levels are almost undetectable in adult tissues. Nonetheless, HMGA levels are frequently upregulated in several different neoplasms, being their overexpression associated with tumor poor prognosis [16]. In this sense, a comprehensive meta-analysis has recently reported the significant impact of high levels of HMGA2 mRNA and/or protein levels on the diminution of cancer patients' overall survival, e.g., of patients affected by renal cell carcinoma, head and neck cancer, hepatocellular carcinoma, and pancreatic ductal adenocarcinoma [17]. HMGA proteins play an important role in cell transformation mainly due to their ability in controlling the expression of genes involved in cell proliferation and invasion control [16]. Contrary to the well-identified upregulation of HMGA in neoplasms and its role in tumorigenesis, the mechanisms underlying their mRNA and protein level expression are not yet completely understood. Lately, it has been reported that epigenetic mechanisms may play an important role in this process. Specifically, long noncoding RNAs and miRNAs have been demonstrated to regulate both HMGA1 and HMGA2 expression. Additionally, two HMGA pseudogenes have been recently identified and described as capable of modulating HMGA protein levels since they act as a decoy, hampering their degradation by miRNAs $[18,19]$. The involvement of HMGA1 and HMGA2 in tumorigenesis has been largely reported along the last years, once the aberrant expression of these genes possesses implications not only in the tumor biology but also in cancer management, characterizing HMGA genes as potential diagnosis and prognosis biomarkers for several different tumors [13].

\section{HMGA and Esophageal Cancer}

In the last years, the growing knowledge in cancer biology promoted the development of new tools for early detection and more specific treatment of most cancer types. The technological revolution, mainly represented by large-scale gene expression analysis, and the development of selective target drugs have been figuring as new and promising strategies in the management of the disease [20]. On the other hand, esophageal cancer (EC) remains poorly impacted by these new approaches, a fact that could be partially explained due to the relative unexploited biology of this tumor [11]. In this sense, despite the scientific evolution experienced during the last years, EC is still characterized as a highly lethal tumor that presents a disappointing scenario where the late detection and poor prognosis are associated with no increment in the therapeutic strategies available for this cancer type [21]. In this way, the identification of new biological markers and the understanding of their role in EC development and progression comprise a fundamental step to a deeper knowledge of the disease. In addition, EC is mainly represented by two main histopathological types: esophageal squamous cell carcinoma (ESCC) and esophageal adenocarcinoma (EAC) [11]. These two EC subtypes largely differ in several aspects that include etiological factors, geographic location, and molecular alterations [22]. In this way, it is known that ESCC and EAC present alterations that are not necessarily shared and include EGFR amplification, differential patterns of DNA methylation, microRNA expression, and alterations in genes particularly involved in the regulation of the cell cycle [23]. On the contrary, it is well established that the most frequent alterations in both ESCC and EAC are the mutations in the tumor suppressor gene TP53 (tumor protein p53) that is present in about $70-80 \%$ of EC cases [24]. Therefore, the improvement in EC molecular landscape knowledge is a mandatory step to upgrade the management of patients affected by this tumor. Moreover, this notion is particularly relevant along the evolution of EAC, once this histotype displays a relatively consolidated natural history, being the influence of obesity, gastroesophageal reflux disease (GERD), and Barrett's esophagus (BE) well-known risk factors associated with EAC genesis and progression [23]. BE is a metaplasia characterized by the replacement of the squamous epithelium by a columnar intestinal-like epithelium that has been associated with an increasing risk of EAC development [6]. In fact, the search for alterations in Barrett's metaplasia has been faced as the "lost link" along the development of EAC, since this condition often precedes the onset of the malignancy [25]. Accordingly, increased expression of HMGA1 along BE's progression has already been reported. Additionally, HMGA1 expression was positive in all BE cases that displayed high-grade dysplasia, whether its expression was barely detected in the BE patients' samples without dysplasia or with low-grade dysplasia [6]. Thus, HMGA1 expression pattern, represented by the increase in its expression along $\mathrm{BE}$ progression, has been suggested as an important approach for early EAC detection, through the screening of $\mathrm{BE}$ patients. Accordingly, some other biomarkers, such as TP53, CDKN2A, CTNNB1, CDH1, GPX3, 
and NOX5, among others have also been described to this end recently [26]. Additionally, converging with the importance of obesity as a crucial and independent risk factor for both $\mathrm{BE}$ and EAC development $[23,26]$, it is largely known that HMGA1 plays a crucial role in the adipogenesis [27]. Therefore, the investigation of HMGA1 expression in obese patients could reveal important insights about EAC evolution, since obesity has been reported as an independent risk factor for EAC development, even in the absence of GERD, that is the main inductor of the BE [28]. In accordance with this hypothesis, HMGA1 could be increasingly expressed along tumor evolution. Our group has already reported that HMGA1 is highly expressed in EAC, but not HMGA2 [29]. Interestingly, and on the opposite way, we observed that HMGA1 expression was almost absent in ESCC samples, but not HMGA2, which expression was markedly positive [29]. In accordance with the difference in HMGA gene and protein expression patterns depending on EC histotype, Toyozumi and colleagues showed a significative difference in HMGA1 expression between EAC and paired healthy esophageal tissue samples [30]. Nevertheless, in this study, the potential of HMGA1 expression as a diagnostic biomarker was not evaluated, as well as HMGA2 expression in the EAC samples investigated. Otherwise, these observations are quite interesting, particularly when seen under the prism of the major differences (etiological factors, geographic location, and molecular alterations) that characterize the two EC main histotypes. In fact, HMGA2 differential expression has been mainly, but not exclusively, associated with squamous tumors [31-33], being its expression related with several aspects of tumor evolution, particularly, invasion and metastasis [34]. As a matter of fact, during the progression of carcinomas, adhesion loss, extracellular matrix remodeling, and cytoskeleton reorganization promoted by epithelial mesenchyme transition (EMT) increase the malignant cell migration and invasion [35]. Besides consisting of a hallmark of tumor progression, EMT is also classically activated during healing process by several cytokines, particularly by transforming growth factor $\beta$ (TGF- $\beta$ ) [36]. In an elegant study, Thualt and colleagues showed that canonical TGF- $\beta$ signaling pathway activates SMAD proteins, ending up in the induction of the expression of transcription factors involved with EMT process [37]. In the same study, the authors demonstrated that EMT activated by TGF- $\beta$ cannot occur in the absence of HMGA2, due to the fact that the activity of EMT transcription factors Twist, Slug, and specially Snail, was dependent on HMGA2 expression. Since then, several studies have reported that HMGA2 plays an essential role in EMT activation [38-41]. Moreover, the clinical relevance of HMGA2 expression levels has also been reported in a study which showed a significant association between its increased expression and occurrence of lymph node metastasis [42]. Contrarily, HMGA1 has been primarily involved in the genesis and evolution of adenocarcinomas, displaying a promising role not only as a diagnostic and prognostic biomarker but also by participating in the control of important malignant hallmarks, such as cell cycle, proliferation, and apoptosis, through the regulation of the expression of key genes (cyclin A1, Rb, p53, and Bax) involved in this process [43]. Finally, it was recently reported by our group that HMGA1, but not HMGA2, is a promising biomarker for endometrial adenocarcinoma development [44]. Furthermore, we demonstrated that HMGA2 displays an interesting potential in the detection of the larynx squamous carcinoma and HMGA1 expression does not seem to play any significant role in laryngeal carcinogenesis [33].

\section{HMGA and Gastric Cancer}

Gastric cancer (GC) is historically a high incidence cancer. Around 70 years ago, GC has been figured as the most frequent type of cancer. Its frequency has diminished along the years; nevertheless, it still ranks as the fifth most incidence tumor worldwide, among men and women [45]. The exact causes for the decrease in GC cases along the years have not been totally elucidated yet; however, an improvement in food maintenance practices probably played an important role in this process [46]. Currently, the incidence of the gastric cancer is particularly high in the Asian countries, especially in Japan. Additionally, similarly to EC, GC development is also strongly related to the exposition of the etiological factors associated with its genesis, particularly, the amount of salt present in the diet and Helicobacter pylori infection [47]. Moreover, GC presents a high mortality rate, being a very poor prognosis tumor and responsible for 782,685 deaths worldwide last year [48]. The intestinal and diffuse types comprise the main GC histopathological subtypes, being well established that the intestinal type is the most frequently detected subtype and mainly associated with Helicobacter pylori infection, while the diffuse subtype occurs predominantly in young and female individuals [47]. Recently, enhanced efforts allowed the identification of the molecular alterations harbored by gastric tumors and, further, made possible its classification into four different molecular subtypes, according to the main alterations present within the different tumors $[49,50]$. The molecular GC classification may be valuably employed together with its histopathology. In this sense, some molecular alterations frequently found in GC occur almost exclusively in one of the subtypes: for instance, amplification of EGFR, ERBB-2, and MET and TP53 mutations occur predominantly in the intestinal subtype, whereas $\mathrm{CDH} 1$ and RHOA mutations are mainly detected in the diffuse subtype $[49,50]$. On the other hand, GC evolution has been classically associated with a multistep sequence of histopathological alterations, which include the development of gastritis, atrophic gastritis, and intraepithelial neoplasia [51]. In this respect, it was previously reported that the microRNA Let7 expression is progressively lost during GC development, since a downregulation of Let7 expression is observed in autoimmune gastritis patients, a condition that is strongly associated with the development of gastric mucosal atrophy and GC carcinogenesis. Furthermore, it was also observed that Let7 expression is decreased in patients infected with Helicobacter pylori, being its expression restored upon infection eradication [45]. As a matter of fact, Let7 microRNA expression deregulation has been related with the development of several cancer types, including GC [52]. 
Moreover, even though the mechanisms involved in HMGA member gene expression regulation have not yet been completely elucidated, it has been shown that HMGA2 expression could be directly regulated by the members of Let7 microRNA family [53]. Furthermore, Let7 has also been implicated in HMGA2 biology, once its in vitro overexpression is able to drastically reduce HMGA2 expression, which culminates in the abrogation of EMT, an important phenomenon occurring during carcinogenesis, in which HMGA2 is known to play a crucial role $[38,54]$. Additionally, the axis Let7/HMGA2 seems to be important not only for the initiation of GC but also for its recurrence, since Takahashi and colleagues demonstrated that the remaining gastric mucosal areas following surgical resection, which are highly related to GC recurrence, display a significant reduction in Let7a expression concomitantly with an increase in the EMT transcription factor Snail [55]. Furthermore, the authors reported that Let7a inhibition in well-differentiated GC cell lines was able to increase HMGA2 and Snail expression, thus corroborating the key role of Let7a and HMGA2 in triggering EMT during GC carcinogenesis [55]. Moreover, diet is already known as an important etiological factor associated with GC development, being the intake of nitrate-enriched food associated with the generation of mutagenic/carcinogenic compounds, such as nitrosamines and nitrosamides [56]. In this sense, in a study in which the classic model of gastric carcinogenesis, by using $N$-ethyl- $N$-nitrosourea (ENU), was employed, a significant reduction in Let7b expression was observed after 15 days of ENU treatment [57], being the importance of this member of Let family in the regulation of HMGA2 expression, demonstrated in an in vitro model of lung cancer progression [58]. In addition, the relationship between HMGA2 and nitrosamine compounds might be even deeper, once HMGA2 was demonstrated to be a key player in DNA damage repair induced by $N$-methyl- $N$-nitrosourea (MNU) [59]. Thus, due to the close association of HMGA2 with the etiological conditions associated with GC development (Helicobacter pylori infection, mucosal metaplastic transformation, and nitrosamine exposure), one could state that HMGA2 plays a remarkable role in gastric carcinogenesis. However, HMGA2 overexpression could also be figured as a potential prognostic biomarker for GC progression, since the deregulation of its expression has been associated with several clinical pathological parameters which include vasculogenic mimicry during GC progression and disease recurrence $[7,60,61]$. In this sense, a recent meta-analysis reported a significant association between increased levels of HMGA2 and later tumor stage, lymph node metastasis, vascular invasion, and diminished overall survival of gastric cancer patients, thus pointing out its potential role as a prognostic biomarker for gastric tumors [62]. Finally, oppositely to other GI tumors, HMGA family expression biomarker potential in GC seems to be restricted to HMGA2 due to the fact that only one study reported the HMGA1 overexpression in GC, otherwise, no association between HMGA1 and any clinical pathological parameter nor disease onset was observed [7].

\section{HMGA and Colorectal Cancer}

Colorectal cancer (CRC) has been figured as one of the most prevalent solid tumors, occupying the third position in incidence and the second in mortality, in both sexes worldwide [48]. Hopefully, probably due to the highest prevalence rates of this tumor occurring in the western population, particularly in developed countries, the management of the disease has been improved in the last years, compared with other tumors, such as esophageal cancer [63]. Moreover, the high incidence rates of CRC in western countries are mainly related to their lifestyle, which includes hypercaloric diet, high intake of red meat, and tobacco and alcohol consumption [64]. Additionally, intestine inflammatory pathologies, like Crohn's disease, and especially, ulcerative colitis, represent the major clinical conditions related to CRC development [64]. CRC presents a wellestablished natural history, not only regarding the main risk factors associated with its development but also in what concerns the main molecular alterations harbored by these tumors [65]. In this sense, genomic instability in CRC is represented by distinct sets of molecular alterations that allowed the molecular subclassification of these tumors into four groups [65]. Therefore, the chromosomal instability (CIN) group is the most representative one and responds for nearly $85 \%$ of all CRC cases, being particularly characterized by the presence of mutations in the tumor suppressors TP53 and $A P C$ [66]. Furthermore, the remaining groups are related to other molecular events, such as microsatellite instability, methylation pattern, and DNA damage repair [67]. In addition, this well-defined scenario, which characterizes CRC, also reveals that among GI tumors, CRC is the one which possesses the best-characterized relationship with HMGA family members. In this way, it has been previously reported that HMGA1 is expressed low in healthy, nontumor colorectal mucosa, whereas its expression gradually increments along CRC evolution [4]. In this study, the authors observed that HMGA1 expression progressively increases from adenoma with mild atypia to adenoma with severe atypia, and, finally, to CRC, thus showing that the alterations in the HMGA1 expression pattern comprise an initial event along malignant evolution, thus indicating a strong potential of HMGA1 expression levels to be used in CRC early detection [4]. In this sense, it was demonstrated that HMGA1 overexpression was able to induce the emergence of polyps in a transgenic mouse model [68], thus reinforcing the relationship between HMGA1 expression deregulation and the early steps of CRC carcinogenesis, since polyps represent a precancerous lesion that, when surgically resected, prevents CRC development [68]. Additionally, by using the same murine model and proteomic approach, Williams and colleagues showed that HMGA1 overexpression was able to alter fatty acids biosynthesis and to decrease taurocholic acid, being these results also observed in CRC tissue [69]. Thus, these data seem very interesting, once the alterations in both pathways have been demonstrated to be related to the neoplastic transformation [70]. Ultimately, it was recently showed that CRC patients displayed high levels of HMGA1 protein in the blood, 
compared to healthy individuals, thus revealing a potential use of HMGA1 expression levels as a noninvasive CRC diagnostic biomarker [71]. In addition, it was observed that aberrant expression of HMGA2 could also be efficiently detected in the blood of CRC patients, compared to healthy individuals [72]. However, the methods employed for HMGA1 and HMGA2 expression measurement in the blood of the individuals were completely distinct: to evaluate HMGA1 expression, a monoclonal antibody-based platform was used, while HMGA2 detection was performed by using cell-free circulating RNA approach. However, despite the differences regarding the technical principle of the two methods, both approaches could represent an improvement in the management of CRC patients in the future, particularly in its detection, since HMGA2 is also overexpressed in CRC tissue, in addition to its association with patients' poor prognosis [73, 74]. Furthermore, Fan and colleagues detected a low expression of miR-543, an HMGA2 regulator microRNA, in CRC samples. Moreover, the authors also showed a downregulation of miR-543 in a mice model of colitis-associated colon cancer, which consists in a pathological condition associated with CRC development [75]. Therefore, as well demonstrated for HMGA1, it seems that the aberrant expression of HMGA2 might be involved in the early steps of CRC development. Additionally, as cited before, alcohol consumption is considered an important risk factor for CRC development [64]. In this sense, it was already reported that the injury induced by ethanol is able to drastically reduce the expression of several microRNAs belonging to Let7 family, whose members are known to downregulate HMGA2 expression [64]. Finally, obesity is considered one of the main conditions associated with CRC development. The increase in calorie consumption in developing countries has been associated with an exponentially growing number of cancer cases, including CRC [76]. Furthermore, it has been reported that HMGA1 and HMGA2 play a dual role in the adipogenesis, being the expression of these genes associated with fat tissue development $[27,77]$. Therefore, it is known that HMGA1 expression impairs adipocyte differentiation, while HMGA2 expression induces adipocyte differentiation, being these effects due to a down- or upregulation exerted by HMGA1/ HMGA2 on key genes involved in adipogenesis [27, 77]. In this way, HMGA1 and HMGA2 altered expression could also be envisaged as a biomarker panel to track CRC risk patients, since, under obesity condition, these genes exhibit an antagonistic expression pattern.

\section{Conclusion}

GI tumors represent a heterogeneous group of neoplasms concerning their natural history and molecular alterations harbored. Nevertheless, these tumors share very high incidence and mortality rates worldwide and patients' poor prognosis. Thus, additionally to patients' suffering, GI tumors heavily impact public health systems worldwide. Therefore, the increment in the molecular knowledge of these malignancies represents an unique opportunity to expand the portfolio of strategies for prevention, diagnosis, and treatment. Furthermore, it shall be the best option to revert the current dark scenario of GI tumors and, thus, represents a mandatory step to improve the management of GI cancers, being HMGA genes and proteins a convergence point in the complex landscape of GI tumors.

\section{Conflicts of Interest}

The authors declare that they have no conflicts of interest.

\section{Acknowledgments}

The authors would like to thank the Conselho Nacional de Desenvolvimento Científico e Tecnológico (CNPq, Brazil) and Fundação de Amparo à Pesquisa Carlos Chagas Filho (FAPERJ, Brazil) for supporting Brazilian research.

\section{References}

[1] A. Runyan, J. Banks, and D. S. Bruni, "Current and future oncology management in the United States," Journal of Managed Care \& Specialty Pharmacy, vol. 25, no. 2, pp. 272-281, 2019.

[2] C. Pauli, B. D. Hopkins, D. Prandi et al., "Personalized in vitro and in vivo cancer models to guide precision medicine," Cancer Discovery, vol. 7, no. 5, pp. 462-477, 2017.

[3] A. Fusco and M. Fedele, "Roles of HMGA proteins in cancer," Nature Reviews Cancer, vol. 7, no. 12, pp. 899-910, 2007.

[4] N. Abe, T. Watanabe, M. Sugiyama et al., "Determination of high mobility group I (Y) expression level in colorectal neoplasias: a potential diagnostic marker," Cancer Research, vol. 59, no. 6, pp. 1169-1174, 1999.

[5] G. Chiappetta, G. Manfioletti, F. Pentimalli et al., "High mobility group HMGI (Y) protein expression in human colorectal hyperplastic and neoplastic diseases," International Journal of Cancer, vol. 91, no. 2, pp. 147-151, 2001.

[6] X. Chen, J. Lechago, A. Ertan et al., "Expression of the high mobility group proteins HMGI (Y) correlates with malignant progression in Barrett's metaplasia," Cancer Epidemiology Biomarkers \& Prevention, vol. 13, no. 1, pp. 30-33, 2004.

[7] K.-H. Jun, J.-H. Jung, H.-J. Choi, E.-Y. Shin, and H.-M. Chin, "HMGA1/HMGA2 protein expression and prognostic implications in gastric cancer," International Journal of Surgery, vol. 24, pp. 39-44, 2015.

[8] M. M. Binabaj, A. Soleimani, F. Rahmani et al., "Prognostic value of high mobility group protein A2 (HMGA2) overexpression in cancer progression," Gene, vol. 706, pp. 131-139, 2019.

[9] World Cancer Report 2014, "Cancer by organ sites," in World Cancer Reports, B. W. Stewart and C. P. Wild, Eds., WHO, Geneva, Switzerland, 2014.

[10] C. Figueiredo, M. C. Camargo, M. Leite, E. M. FuentesPananá, C. S. Rabkin, and J. C. Machado, "Pathogenesis of gastric cancer: genetics and molecular classification," Current Topics in Microbiology and Immunology, vol. 400, pp. 277304, 2017.

[11] M. Sohda and H. Kuwano, "Current status and future prospects for esophageal cancer treatment," Annals of Thoracic and Cardiovascular Surgery, vol. 23, no. 1, pp. 1-11, 2017.

[12] E. F. Onyoh, W. F. Hsu, L. C. Chang, Y. C. Lee, M. S. Wu, and H. M. Chiu, "The rise of colorectal cancer in Asia: epidemiology, screening, and management," Current Gastroenterology Reports, vol. 21, no. 8, p. 36, 2019. 
[13] S. N. Shah and L. M. Resar, "High mobility group A1 and cancer: potential biomarker and therapeutic target," Histology and Histopathology, vol. 27, no. 5, pp. 567-579, 2012.

[14] T. Cui and F. Leng, "Specific recognition of AT-rich DNA sequences by the mammalian high mobility group protein AT-hook 2: a SELEX study," Biochemistry, vol. 46, no. 45, pp. 13059-13066, 2007.

[15] R. Reeves, "Nuclear functions of the HMG proteins," Biochimica et Biophysica Acta (BBA)—Gene Regulatory Mechanisms, vol. 1799, no. 1-2, pp. 3-14, 2010.

[16] M. Fedele and A. Fusco, "HMGA and cancer," Biochimica et Biophysica Acta (BBA)-Gene Regulatory Mechanisms, vol. 1799, no. 1-2, pp. 48-54, 2010.

[17] B. Huang, J. Yang, Q. Cheng et al., "Prognostic value of HMGA2 in human cancers: a meta-analysis based on literatures and TCGA datasets," Frontiers in Physiology, vol. 9, pp. 776-787, 2018.

[18] F. Esposito, M. De Martino, M. G. Petti et al., "HMGA1 pseudogenes as candidate proto-oncogenic competitive endogenous RNAs," Oncotarget, vol. 5, no. 18, pp. 8341-8354, 2014.

[19] F. Esposito, M. De Martino, D. D’Angelo et al., "HMGA1pseudogene expression is induced in human pituitary tumors," Cell Cycle, vol. 14, no. 9, pp. 1471-1475, 2015.

[20] A. Palumbo Jr., N. D. O. Da Costa, M. H. Bonamino, L. F. Pinto, and L. E. Nasciutti, "Genetic instability in the tumor microenvironment: a new look at an old neighbor," Molecular Cancer, vol. 14, no. 1, p. 145, 2015.

[21] G. Roshandel, A. Nourouzi, A. Pourshams, S. Semnani, S. Merat, and M. Khoshnia, "Endoscopic screening for esophageal squamous cell carcinoma," Archives of Iranian Medicine, vol. 16, pp. 351-357, 2013.

[22] N. M. da Costa, S. C. Soares Lima, T. de Almeida Simão, and L. F. Ribeiro Pinto, "The potential of molecular markers to improve interventions throughthe natural history of oesophageal squamous cell carcinoma," Bioscience Reports, vol. 33, no. 4, Article ID e00057, 2013.

[23] F. R. Talukdar, M. di Pietro, M. Secrier et al., "Molecular landscape of esophageal cancer: implications for early detection and personalized therapy," Annals of the New York Academy of Sciences, vol. 1434, no. 1, pp. 342-359, 2018.

[24] International Cancer Genome Consortium: http://www.icgc. org/.

[25] S. A. C. McDonald, D. Lavery, N. A. Wright, and M. Jansen, "Barrett oesophagus: lessons on its origins from the lesion itself," Nature Reviews Gastroenterology \& Hepatology, vol. 12, no. 1, pp. 50-60, 2015.

[26] I. Kalatskaya, "Overview of major molecular alterations during progression from Barrett's esophagus to esophageal adenocarcinoma," Annals of the New York Academy of Sciences, vol. 1381, no. 1, pp. 74-91, 2016.

[27] A. Arce-Cerezo, M. García, A. Rodríguez-Nuevo et al., "HMGA1 overexpression in adipose tissue impairs adipogenesis and prevents diet-inducedobesity and insulin resistance," Scientific Reports, vol. 5, no. 1, p. 14487, 2015.

[28] A. P. Thrift, N. J. Shaheen, M. D. Gammon et al., "Obesity and risk of esophageal adenocarcinoma and Barrett's esophagus: a Mendelian randomization study," JNCI: Journal of the National Cancer Institute, vol. 106, no. 11, 2014.

[29] A. Palumbo Jr., N. M. Da Costa, F. Esposito et al., "HMGA2 overexpression plays a critical role in the progression of esophageal squamous carcinoma," Oncotarget, vol. 7, no. 18, pp. 25872-25884, 2016.
[30] T. Toyozumi, I. Hoshino, M. Takahashi et al., "Fra-1 regulates the expression of HMGA1, which is associated with a poor prognosis in human esophageal squamous cell carcinoma," Annals of Surgical Oncology, vol. 24, no. 11, pp. 3446-3455, 2017.

[31] C. Hebert, K. Norris, M. A. Scheper, N. Nikitakis, and J. J. Sauk, "High mobility group A2 is a target for miRNA-98 in head and neck squamous cell carcinoma," Molecular Cancer, vol. 6, no. 1, pp. 5-12, 2007.

[32] K. A. Sterenczak, A. Eckardt, A. Kampmann et al., "HMGA1 and HMGA2 expression and comparative analyses of HMGA2, Lin28 and let-7 miRNAs in oral squamous cell carcinoma," BMC Cancer, vol. 14, no. 1, pp. 694-702, 2014.

[33] A. Palumbo Jr., M. De Martino, F. Esposito et al., "HMGA2, but not HMGA1, is overexpressed in human larynx carcinomas," Histopathology, vol. 72, no. 7, pp. 1102-1114, 2018.

[34] A. Palumbo Júnior, N. M. Da Costa, F. Esposito, A. Fusco, and L. F. R. Pinto, "High mobility Group A proteins in esophageal carcinomas," Cell Cycle, vol. 15, no. 18, pp. 2410-2413, 2016.

[35] F. Perlikos, K. J. Harrington, and K. N. Syrigos, "Key molecular mechanisms in lung cancer invasion and metastasis: a comprehensive review," Critical Reviews in Oncology/Hematology, vol. 87, no. 1, pp. 1-11, 2013.

[36] S. Lindsey and S. A. Langhans, "Crosstalk of oncogenic signaling pathways during epithelial mesenchymal transition," Frontiers in Oncology, vol. 4, pp. 1-10, 2014.

[37] S. Thuault, U. Valcourt, M. Petersen, G. Manfioletti, C.-H. Heldin, and A. Moustakas, "Transforming growth factor- $\beta$ employs HMGA2 to elicit epithelial-mesenchymal transition," The Journal of Cell Biology, vol. 174, no. 2, pp. 175-183, 2006.

[38] S. Thuault, E.-J. Tan, H. Peinado, A. Cano, C.-H. Heldin, and A. Moustakas, "HMGA2 and Smads co-regulate SNAIL1 expression during induction of epithelial-to-mesenchymal transition," Journal of Biological Chemistry, vol. 283, no. 48, pp. 33437-33446, 2008.

[39] S. Watanabe, Y. Ueda, S.-i. Akaboshi, Y. Hino, Y. Sekita, and M. Nakao, "HMGA2 maintains oncogenic RAS-induced epithelial-mesenchymal transition in human pancreatic cancer cells," The American Journal of Pathology, vol. 174, no. 3, pp. 854-868, 2009.

[40] J. Wu, Z. Liu, C. Shao et al., "HMGA2 Overexpression-Induced ovarian surface epithelial transformation is mediated through regulation of EMT genes," Cancer Research, vol. 71, no. 2, pp. 349-359, 2011.

[41] E.-J. Tan, S. Thuault, L. Caja, T. Carletti, C.-H. Heldin, and A. Moustakas, "Regulation of transcription factor twist expression by the DNA architectural protein high mobility group A2 during epithelial-to-mesenchymal transition," Journal of Biological Chemistry, vol. 287, no. 10, pp. 71347145, 2012.

[42] R. Wei, Z. Shang, J. Leng, and L. Cui, "Increased expression of high-mobility group A2: A novel independent indicator of poor prognosis in patients with esophageal squamous cell carcinoma," Journal of Cancer Research and Therapeutics, vol. 12, no. 4, pp. 1291-1297, 2016.

[43] M. Fedele and A. Fusco, "Role of the high mobility group A proteins in the regulation of pituitary cell cycle," Journal of Molecular Endocrinology, vol. 44, no. 6, pp. 309-318, 2010.

[44] A. Palumbo Júnior, V. P. de Sousa, F. Esposito et al., "Overexpression of HMGA1 figures as a potential prognostic factor in endometrioid endometrial carcinoma (EEC)," Genes, vol. 10, no. 5, p. E372, 2019. 
[45] M. Fassan, D. Saraggi, and L. Balsamo, "Let-7c down-regulation in Helicobacter pylori-related gastric carcinogenesis," Oncotarget, vol. 7, no. 4, pp. 4915-4924, 2016.

[46] R. Sitarz, M. Skierucha, J. Mielko, J. Offerhaus, R. Maciejewski, and W. Polkowski, "Gastric cancer: epidemiology, prevention, classification, and treatment," Cancer Management and Research, vol. 10, pp. 239-248, 2018.

[47] B. Hu, N. El Hajj, S. Sittler, N. Lammert, R. Barnes, and A. Meloni-Ehrig, "Gastric cancer: classification, histology and application of molecular pathology," Journal of Gastrointestinal Oncology, vol. 3, no. 3, pp. 251-261, 2012.

[48] F. Bray, J. Ferlay, I. Soerjomataram, R. L. Siegel, L. A. Torre, and A. Jemal, "Global cancer statistics 2018: GLOBOCAN estimates of incidence and mortality worldwide for 36 cancers in 185 countries," CA: A Cancer Journal for Clinicians, vol. 68, no. 6, pp. 394-424, 2018.

[49] The Cancer Genome Atlas Research Network, "Comprehensive molecular characterization of gastric adenocarcinoma," Nature, vol. 513, no. 7517, pp. 202-209, 2014.

[50] S. K. Garattini, D. Basile, M. Cattaneo et al., "Molecular classifications of gastric cancers: novel insights and possible future applications," World Journal of Gastrointestinal Oncology, vol. 9, no. 5, pp. 194-208, 2017.

[51] J. Lage, N. Uedo, M. Dinis-Ribeiro, and K. Yao, "Surveillance of patients with gastric precancerous conditions," Best Practice \& Research Clinical Gastroenterology, vol. 30, no. 6, pp. 913-922, 2016.

[52] T. Ueda, S. Volinia, H. Okumura et al., "Relation between microRNA expression and progression and prognosis of gastric cancer: a microRNA expression analysis," The Lancet Oncology, vol. 11, no. 2, pp. 136-146, 2010.

[53] R. G. Rowe, L. D. Wang, S. Coma et al., "Developmental regulation of myeloerythroid progenitor function by the Lin28b-let-7-HMGA2 axis," The Journal of Experimental Medicine, vol. 213, no. 8, pp. 1497-1512, 2016.

[54] C. Zhu, J. Li, G. Cheng et al., "miR-154 inhibits EMT by targeting HMGA2 in prostate cancer cells," Molecular and Cellular Biochemistry, vol. 379, no. 1-2, pp. 69-75, 2013.

[55] Y. Takahashi, K. Uno, K. Iijima et al., "Acidic bile salts induces mucosal barrier dysfunction through let-7a reduction during gastric carcinogenesis after Helicobacter pylori eradication," Oncotarget, vol. 9, no. 26, pp. 18069-18083, 2018.

[56] M. Dietrich, G. Block, J. M. Pogoda, P. Buffler, S. Hecht, and S. P. Martin, "A review: dietary and endogenously formed N-nitroso compounds and risk of childhood brain tumors," Cancer Causes \& Control, vol. 16, no. 6, pp. 619-635, 2005.

[57] Z. Li, W. S. Branham, S. L. Dial et al., "Genomic analysis of microRNA time-course expression in liver of mice treated with genotoxic carcinogen N-ethyl-N-nitrosourea," $B M C$ Genomics, vol. 11, no. 1, p. 609, 2010.

[58] Y. S. Lee and A. Dutta, "The tumor suppressor microRNA let7 represses the HMGA2 oncogene," Genes \& Development, vol. 21, no. 9, pp. 1025-1030, 2007.

[59] R. Fujikane, K. Komori, M. Sekiguchi, and M. Hidaka, "Function of high-mobility group A proteins in the DNA damage signaling for the induction of apoptosis," Scientific Reports, vol. 6, no. 1, 2016.

[60] D. Kong, G. Su, L. Zha et al., "Coexpression of HMGA2 and Oct4 predicts an unfavorable prognosis in human gastric cancer," Medical Oncology, vol. 31, no. 8, p. 130, 2014.

[61] J. Sun, B. Sun, R. Sun et al., "HMGA2 promotes vasculogenic mimicry and tumor aggressiveness by upregulating Twist 1 in gastric carcinoma," Scientific Reports, vol. 7, no. 1, p. 2229, 2017.
[62] J. Zhu, H. Wang, S. Xu, and Y. Hao, "Clinicopathological and prognostic significance of HMGA2 overexpression in gastric cancer: a meta-analysis," Oncotarget, vol. 8, no. 59, pp. 100478-100489, 2017.

[63] B. A. Weinberg, J. L. Marshall, and M. E. Salem, "The growing challenge of young adults with colorectal cancer," Oncology, vol. 31, no. 5, pp. 381-389, 2017.

[64] L. Roncucci and F. Mariani, "Prevention of colorectal cancer: how many tools do we have in our basket?," European Journal of Internal Medicine, vol. 26, no. 10, pp. 752-756, 2015.

[65] M. F. Müller, A. E. K. Ibrahim, and M. J. Arends, "Molecular pathological classification of colorectal cancer," Virchows Archiv, vol. 469, no. 2, pp. 125-134, 2016.

[66] X. Sagaert, A. Vanstapel, and S. Verbeek, "Tumor heterogeneity in colorectal cancer: what do we know so far?"” Pathobiology, vol. 85, no. 1-2, pp. 72-84, 2018.

[67] L. D. Wood, D. W. Parsons, S. Jones et al., "The genomic landscapes of human breast and colorectal cancers," Science, vol. 318, no. 5853, pp. 1108-1113, 2007.

[68] A. Belton, A. Gabrovsky, Y. K. Bae et al., "HMGA1 induces intestinal polyposis in transgenic mice and drives tumor progression and stem cell properties in colon cancer cells," PLoS One, vol. 7, no. 1, Article ID e30034, 2012.

[69] M. D. Williams, X. Zhang, A. S. Belton et al., "HMGA1 drives metabolic reprogramming of intestinal epithelium during hyperproliferation, polyposis, and colorectal carcinogenesis," Journal of Proteome Research, vol. 14, no. 3, pp. 1420-1431, 2015.

[70] D. L. H. Smith, P. Keshavan, U. Avissar, K. Ahmed, and S. D. Zucker, "Sodium taurocholate inhibits intestinal adenoma formation in $\mathrm{APC}^{\mathrm{Min} /+}$ mice, potentially through activation of the farnesoid X receptor," Carcinogenesis, vol. 31, no. 6, pp. 1100-1109, 2010.

[71] A. Capo, R. Sepe, G. Pellino et al., "Setting up and exploitation of a nano/technological platform for the evaluation of HMGA1b protein in peripheral blood of cancer patients," Nanomedicine: Nanotechnology, Biology and Medicine, vol. 15, no. 1, pp. 231-242, 2019.

[72] S. Sahengbieke, J. Wang, X. Li, Y. Wang, M. Lai, and J. Wu, "Circulating cell-free high mobility group AT-hook 2 mRNA as a detection marker in the serum of colorectal cancer patients," Journal of Clinical Laboratory Analysis, vol. 32, no. 4, Article ID e22332, 2018.

[73] X. Wang, X. Liu, A. Y.-J. Li et al., "Overexpression of HMGA2 promotes metastasis and impacts survival of colorectal cancers," Clinical Cancer Research, vol. 17, no. 8, pp. 2570-2580, 2011.

[74] C. Rizzi, P. Cataldi, A. Iop et al., "The expression of the highmobility group A2 protein in colorectal cancer and surrounding fibroblasts is linked to tumor invasiveness," Human Pathology, vol. 44, no. 1, pp. 122-132, 2013.

[75] C. Fan, Y. Lin, Y. Mao et al., "MicroRNA-543 suppresses colorectal cancer growth and metastasis by targeting KRAS, MTA1and HMGA2," Oncotarget, vol. 7, no. 16, pp. 2182521839, 2016.

[76] F. K. Tabung, L. S. Brown, and T. T. Fung, "Dietary patterns and colorectal cancer risk: a review of 17 Years of evidence (2000-2016)," Current Colorectal Cancer Reports, vol. 13, no. 6, pp. 440-454, 2017.

[77] Y. Xi, W. Shen, L. Ma et al., "HMGA2 promotes adipogenesis by activating C/EBP $\beta$-mediated expression of PPAR $\gamma$," Biochemical and Biophysical Research Communications, vol. 472, no. 4, pp. 617-623, 2016. 


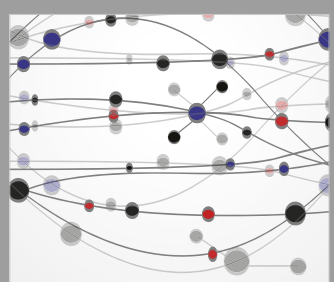

The Scientific World Journal
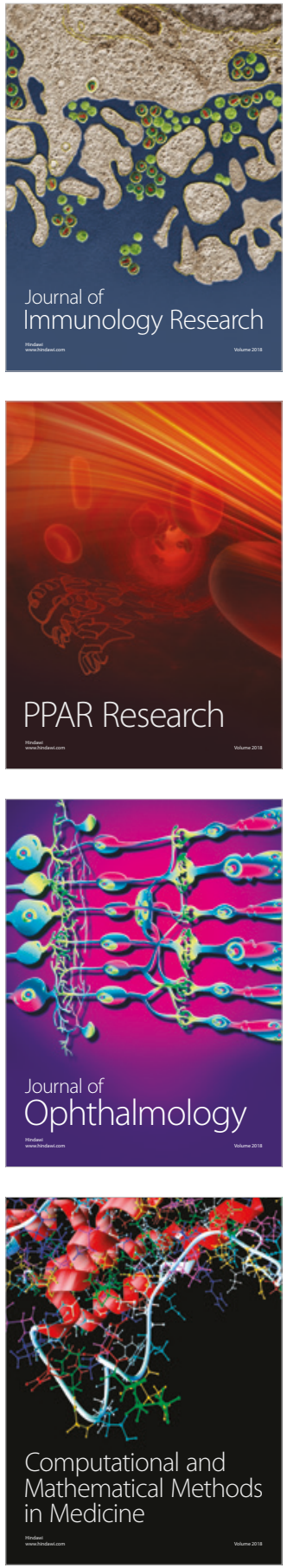

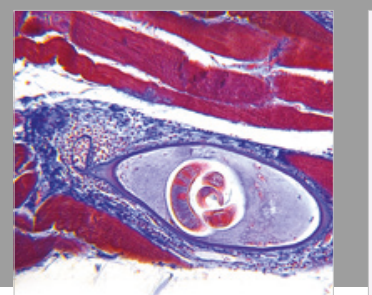

Gastroenterology Research and Practice

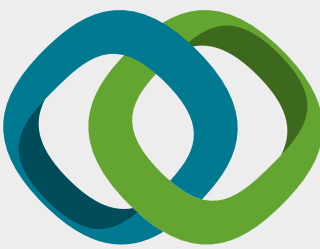

\section{Hindawi}

Submit your manuscripts at

www.hindawi.com
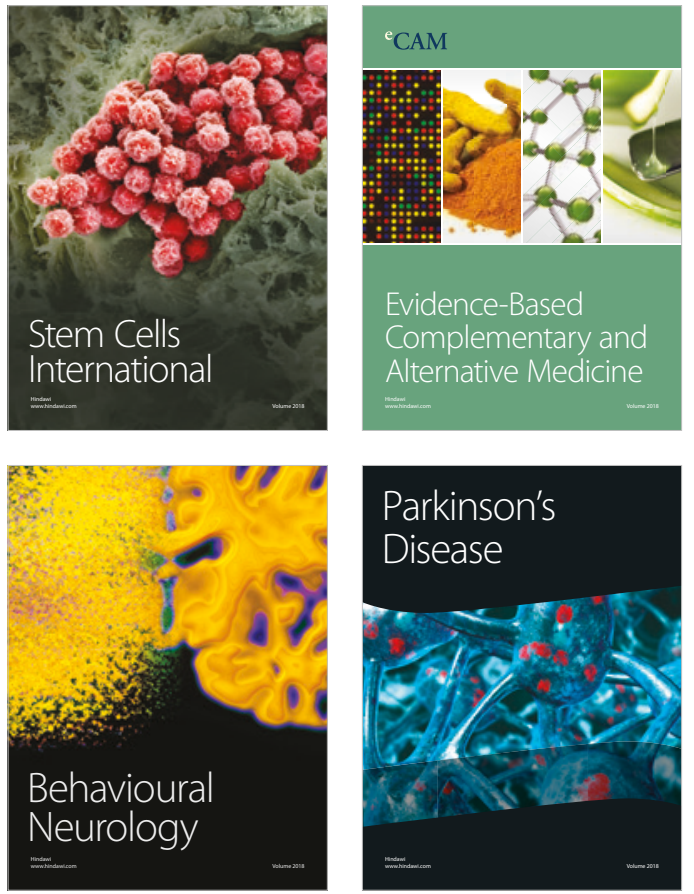

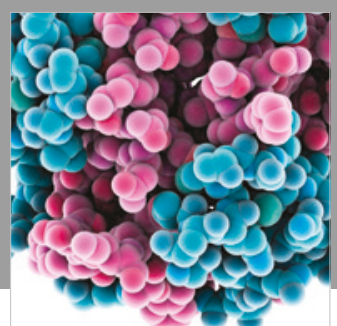

ournal of

Diabetes Research

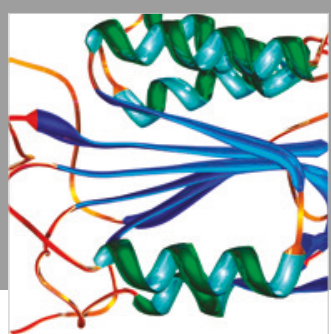

Disease Markers
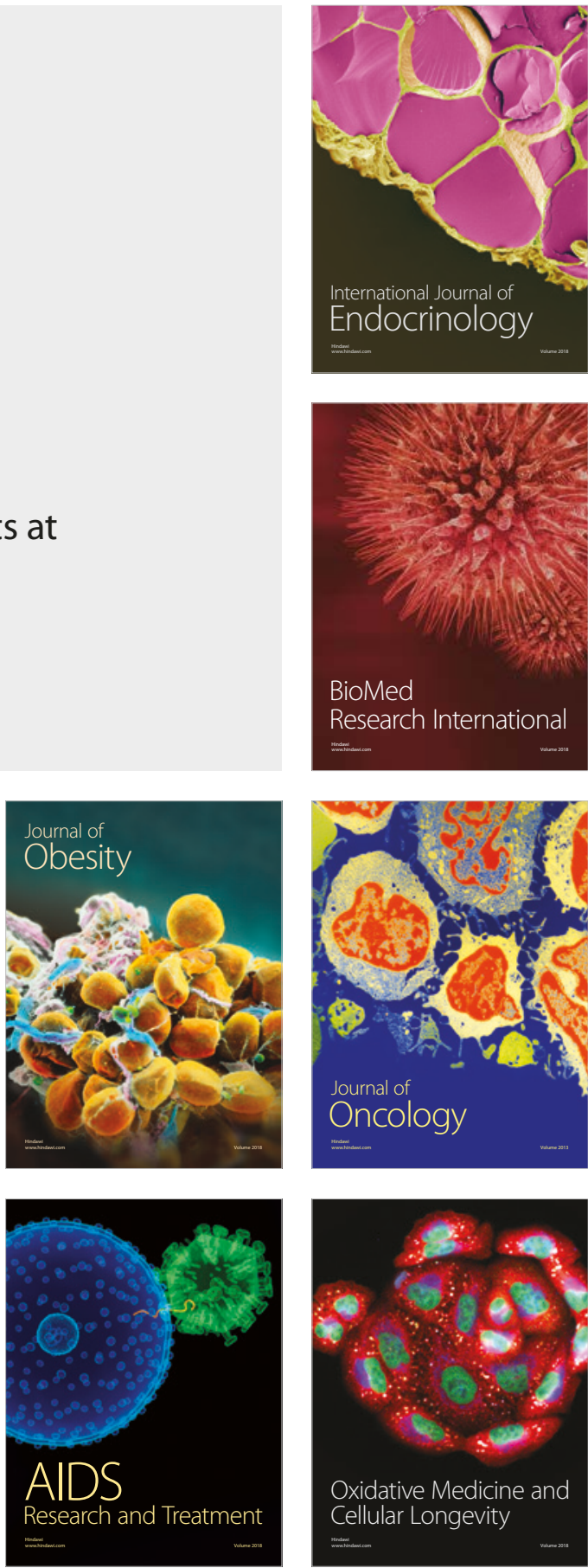